\title{
Grain boundary liquid metal wetting: A synchrotron micro-radiographic investigation
}

\author{
E. Pereiro-López ${ }^{\mathrm{a}, \mathrm{b}, *}$, W. Ludwig ${ }^{\mathrm{a}}$, D. Bellet ${ }^{\mathrm{a}, \mathrm{b}}$, J. Baruchel ${ }^{\mathrm{a}}$ \\ a European Synchrotron Radiation Facility, 6 rue Jules Horowitz, BP 220, 38043 Grenoble, France \\ ${ }^{\mathrm{b}}$ Laboratoire de Génie Physique et Mécanique des Matériaux ENSPG, INPG, BP 46, 38402 Saint-Martin-d Hères cedex, France
}

\begin{abstract}
The penetration of liquid metals into the grain boundaries (GB) of solid metals can lead to severe embrittlement of the solid. Grain boundary wetting, which is the precursor process of liquid metal embrittlement, is still far from being well understood. We report in situ observations of the propagation kinetics of liquid metal into the GB of bicrystals and polycrystals. The experiments are performed using synchrotron radiation micro-radiography. This imaging technique is well adapted for the in situ non-destructive bulk characterization of such phenomenon due to suitable spatial and temporal resolutions. Experiments have shown that the Ga penetration front in aluminum bicrystals propagates roughly with linear time dependence, whereas a discontinuous behaviour occurs in polycrystals.
\end{abstract}

(c) 2002 Elsevier Science B.V. All rights reserved.

Keywords: Grain boundary; Grain boundary wetting; Liquid metal embrittlement

\section{Introduction}

When a polycrystalline solid is exposed to a liquid phase, rapid penetration (from $10^{-3}$ up to $100 \mu \mathrm{m} / \mathrm{s}$ ) of the liquid phase along the grain boundaries (GB) takes place in a variety of metallic and ceramic systems [1]. The associated thermodynamic driving force is $\gamma_{\mathrm{GB}}-2 \gamma_{\mathrm{SL}}$, where $\gamma_{\mathrm{GB}}$ and $\gamma_{\mathrm{SL}}$ are the energies of the GB and the solid/liquid interface, respectively. This phenomenon called liquid metal embrittlement [2,3] can

\footnotetext{
${ }^{*}$ Corresponding author. Address: European Synchrotron Radiation Facility, 6 rue Jules Horowitz, BP 220, 38043 Grenoble, France. Tel.: +33-476-882837; fax: +33-476-882252. E-mail address: pereiro@esrf.fr (E. Pereiro-López).
}

entail severe degradation of the mechanical strength of the material. It is of relevance when structural metals are exposed to liquid metal environments under the simultaneous action of either external or internal stresses. The subject has gained renewed interest in recent years, due to international efforts to develop "safe" (under critical) accelerator driven nuclear reactors where liquid metals, such as lead alloy which serves as targets and cooling fluid, should be maintained in contact with a steel reservoir for several years.

In spite of numerous efforts, this mechanism is still far from being well understood. A variety of models have been proposed [2,3], but none of them can fully account for the different experimental aspects of the penetration process. Indeed, several basic questions are still opened [4] such as that 
thermodynamics arguments do not help to understand the formation of macroscopically thick layers since the driving force should not be anymore effective once the penetration layer exceeds a few interatomic distances.

Synchrotron imaging techniques as micro-radiography, micro-tomography (its three-dimensional counterpart), micro-fluorescence or holotomography, allow new ways to carry out experiments in material science [5]. These techniques can be well adapted to investigate in situ grain boundary wetting (GBW) by liquid metals [6]. We report here some micro-radiography in situ observations of the Ga liquid penetration into the $\mathrm{Al}$ GB of bicrystal and polycrystals.

\section{Experimental method and sample preparation}

\subsection{Synchrotron micro-radiography}

Micro-radiography consists of recording conventional projection radiographs based on absorption contrast. The key factors of synchrotron radiation are the very low divergence of the beam, the very high photon flux and the tuneable energy. In such a case, the spatial resolution (limited by the detecting system) is of about $1 \mu \mathrm{m}$ while the high flux enables to record several radiographs per second.

The reported X-ray experiments were carried out at the ID19 beamline of the European Synchrotron Radiation Facility (ESRF, Grenoble,
France). This beamline is devoted to high-resolution imaging. A schematic view of the experimental device is reported in Fig. 1. To get quantitative absorption measurements, the incoming "white' synchrotron radiation is monochromatised using a $\mathrm{Rb}-\mathrm{B}_{4} \mathrm{C}$ multilayer. The detector system consists of a fluorescent screen (LAG:Eu), which transforms X-rays into visible light, the microscope optics and a cooled down $1024^{2}$ or $2048^{2}$ CCD camera. This camera has a fast readout $(0.06$ or $0.24 \mathrm{~s} /$ frame respectively) and low electronic noise. The pixel size is typically of about $1 \mu \mathrm{m}$, then the field of view corresponds to a few $\mathrm{mm}^{2}$, and the typical exposure times for one projection (radiograph) are $0.1-1 \mathrm{~s}$.

\subsection{Sample preparation}

The micro-radiography experiments were performed on two different types of Al samples:

- $99.999 \%$ Al polycrystals. The material was bought cold-drawn (Goodfellow). The $1 \mathrm{~mm}$ thick original samples were cut into plates of about $7 \times 7 \mathrm{~mm}^{2}$. The specimens were then annealed $100 \mathrm{~min}$ at $425{ }^{\circ} \mathrm{C}$. They contain large grains with a typical size of about $1 \mathrm{~mm}$.

- $99.996 \% \mathrm{Al}$ bicrystals with $150^{\circ}$ symmetrical tilt boundary around $\langle 100\rangle$ (general boundary) provided by Ecole des Mines de St. Etienne (M. Biscondi and K. Wolski). $600 \mu \mathrm{m}$ thick bicrystal slices were spark cut from the ingots.

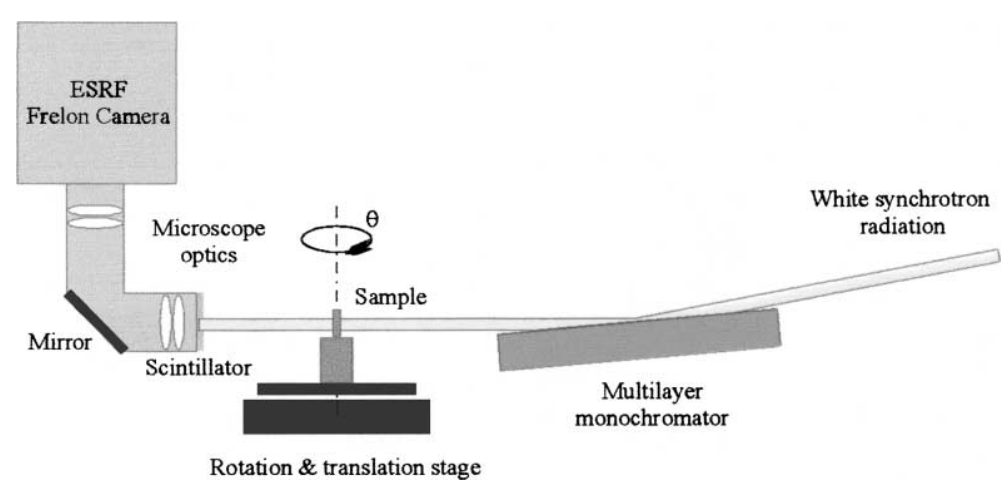

Fig. 1. Schematic view of the experimental ID19 set-up showing the multilayer monochromator, the sample rotation and translation stage and the detector system. 
The sample preparation has to ensure the intimate contact between the Al external surface, previously polished, and the liquid Ga. This was done by scratching the Al sample, which was immediately dipped in liquid nitrogen to avoid the GBW initiation. The sample was fixed on a $\mathrm{Cu}$ plate with a heating element (the Ga melting point being $29.8{ }^{\circ} \mathrm{C}$ ). Two different sample fixations, which gave rise to different stresses, were adopted: either the sample was fixed between two metallic plates, which were screwed together, or glued using silver paint.

\section{Experimental results and discussion}

\subsection{Experimental results obtained on an aluminium bicrystal}

Fig. 2 shows four images out of a sequence of 2000 images (recorded every $1.4 \mathrm{~s}$ ) taken during the GBW of an Al bicrystal. Within the present resolution/sensitivity limit of the set-up, the images clearly exhibit the monotonous penetration and thickening of the Ga liquid film as it propagates along the grain boundary. The darker region corresponds to the projection of the GB, which has been wetted by the liquid $\mathrm{Ga}$ and exhibits different apparent widths directly related to the misorientation between the incoming X-ray beam and the GB plane. These widths are not connected to the Ga thickness itself.

Since the main contrast is the absorption one, we can estimate the thickness of the $\mathrm{Ga}$ layer versus time at different regions along the grain boundary depicted A, B and C in Fig. 2(d). For each one the transmission was measured. If we note as $I_{0}$ the incoming X-ray beam intensity, the transmitted intensity is given by $I_{0} \exp -\left(\mu_{\mathrm{Al}} t_{\mathrm{Al}}+\right.$ $\left.\mu_{\mathrm{Ga}} t_{\mathrm{Ga}}\right)$, where $\mu$ and $t$ are, respectively, the linear attenuation coefficient and the thickness of the crossed $\mathrm{Al}$ or Ga metal along the beam path. The observed transmission variation is directly translated into variations of the Ga layer thickness because the $\mathrm{Al}$ contribution is known from a reference image recorded before the beginning of the GBW process. However we have to consider the projection effect depicted in the inset Fig. 2(e). The transmission value gives us directly an effective thickness $(t)$, which has to be corrected by the
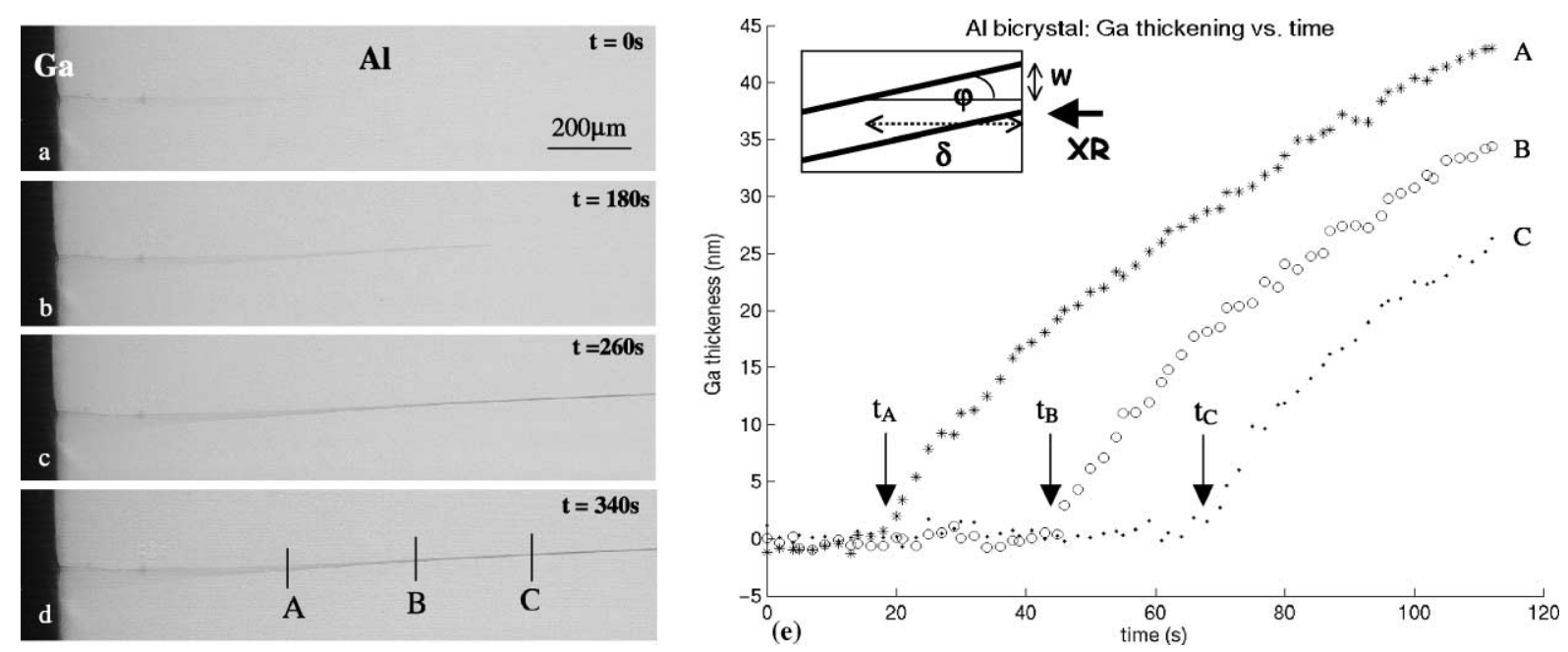

Fig. 2. (a-d) In situ observation of Ga penetration into the grain boundary of an Al bicrystal. The images clearly exhibit the monotonous penetration and thickening of the liquid film as it propagates along the grain boundary. X-rays are perpendicular to the images and the boundary is almost parallel to the beam direction. The different width of the projected GB (see the difference between the left and right parts of the image) is due to the small twist of it, and is not connected to the Ga thickness itself. (e) Evolution of the Ga thickness layer in regions A, B and C (depicted in Fig. 2(d)) versus time. In-set (Fig. 2(e)): incoming X-ray and grain boundary geometry. 
misorientation angle $\varphi$ between the incoming beam direction and the plane of the GB that depends on its geometry. We get the real Ga thickness layer $e(e \approx \varphi t)$.

The slow and monotonous propagation of the Ga front is clearly observed on the plot of Fig. 2(e). The same qualitative behaviour was observed for all the investigated samples and the quantitative results in terms of penetration speed or thickening values were ranging respectively from 1 to $10 \mu \mathrm{m} / \mathrm{s}$ and from 0.2 to $2 \mathrm{~A} / \mathrm{s}$. Higher rates were observed for stressed samples with penetration rates from 10 to $30 \mu \mathrm{m} / \mathrm{s}$ and thickening up to $1 \mathrm{~nm} / \mathrm{s}$.

\subsection{Experimental results obtained on an aluminium polycrystal}

The same type of experiments was performed on Al polycrystals. Fig. 3(a)-(c) present six ra- diographs obtained on one of the samples, at different times during the Ga penetration. We can clearly observe a rather quick Ga penetration along the GB followed by a slow increase of the Ga amount from radiograph (a) and (b), and then, a sudden large contrast increase (see Fig. 3(c)) which corresponds to an important increase in the thickening. As for the bicrystals, we can plot the Ga thickness along the X-ray path versus time in five different regions of the sample correcting the projection effect.

Fig. 3(d) shows the investigated regions, Fig. 3(e)-(g) present the polycrystal behaviour during the wetting process. At the beginning of the $\mathrm{Ga}$ penetration, we can observe a slow increase of the $\mathrm{Ga}$ amount, which propagates from A to, simultaneously, B and C, then to D and finally to E. Unlike for bicrystals, no clear and regular Ga propagation front is observed. Moreover, a
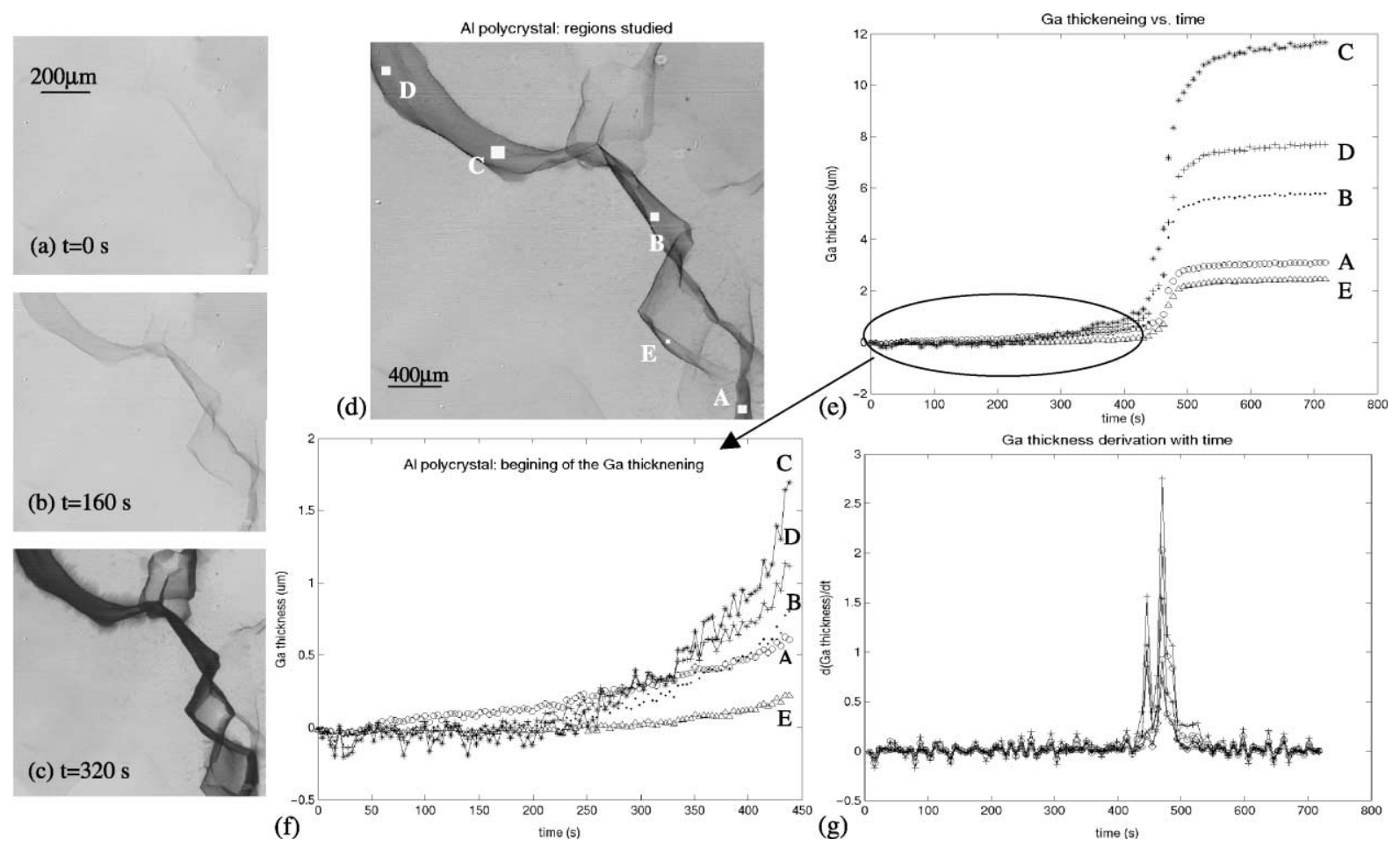

Fig. 3. (a-c) X-ray radiographs of an Al polycrystal at different times during its GB wetting by liquid gallium. (d) Radiograph of an Al polycrystal sample with different regions where the Ga amount was investigated. (e) Ga thickness plotted versus time for the different regions. (f) Zoom of the previous picture enlarged to better see the slow increase of the Ga amount before the discontinuities. (g) The first derivative of the Ga thickness versus time is shown. 
quasi-instantaneous and simultaneous discontinuity in the Ga thickness is recorded for all the regions at least within our time resolution (one radiograph recorded every $0.8 \mathrm{~s})$. The first derivative versus time clearly demonstrates so (Fig. 3(g)). Two discontinuities are observed and we interpreted them as a "mechanical decohesion" of the polycrystal.

As for bicrystals, when investigating many specimens, the Ga amount and the kinetics were higher for stressed samples, but the same general behaviour ruled when GBW took place: discontinuities were always observed. Even if the sample fixation induced no simple and well-defined stresses, the role of the stress on Ga penetration was clearly evidenced.

\section{Discussion}

The experiments related to the bicrystals can be compared with the in situ transmission electron microscopy (TEM) experiments performed on the $\mathrm{Al} / \mathrm{Ga}$ couple as well [7]. The reported features and the speed values are in good agreement with those presented in the present paper. However, the experimental techniques are very different and present different advantages/drawbacks. For instance, the TEM experiments are only related to very thin foils, which perhaps do not exactly correspond to the bulk behaviour of the material. However, this technique gives interesting clues related to the strain fields near the tip of the wetted grain boundary.

The results presented here clearly demonstrate that Ga wetting is very different for well-prepared Al bicrystals and for annealed Al polycrystals. While the Ga penetration was regular and monotonous for the first ones, some important discontinuities were observed for the second type of samples. This stems from at least two phenomena. The Al bicrystals exhibit regular properties along the grain boundary while for polycrystals, the GB are twisted and have complicated shapes. Therefore the last ones have probably non-regular interfacial energies along the GB, as well as for the wetting driving force. The second main difference concerns the presence of stress which is much more probable for polycrystals than for bicrystals.
The other major result of this investigation concerns the influence of the stress on the kinetics, for both types of samples. When stresses (resulting from fixation) are present, larger penetration rates are observed. This is for sure not the first example in the literature relating that stress affects the wetting of a liquid metal, but this is perhaps one of the very first direct observations of the stress effect upon kinetics penetration.

But the present experimental method has clearly two limitations. Our sample fixation does not allow a quantification of the nature and amplitude of the stress. To overcome this limit, a tensile device compatible with the synchrotron micro-radiography experiment is under development. The other limitation arises from the sensitivity of the technique itself. For well-aligned GB such as for bicrystals, one is able to detect Ga films as thin as 10 nanometers. However, for polycrystals, such limit changes drastically to about $100 \mathrm{~nm}$ due to the larger $\varphi$ values. Therefore, we cannot follow the very beginning of the Ga wetting, especially for polycrystals, because we are not able to detect the nanometric precursor film, which is the trigger of this fragilisation process. To detect such nanometric films, we should use Auger spectroscopy [8] or X-ray micro-fluorescence: this latter technique is under development on the ID19 beamline and should be applied in a near future to investigate the liquid metal penetration into solid GB in parallel with micro-radiography but with a higher sensitivity.

\section{Conclusion}

The synchrotron micro-radiography is very well adapted to follow the in situ penetration of liquid Ga along the Al GB. The spatial resolution (about $1 \mu \mathrm{m})$ as well as the temporal resolution (around $1 \mathrm{~s})$ enables the observation of GBW by Ga layers.

The most striking feature is the different penetration behaviour observed in bicrystals and polycrystals. The former exhibit linear Ga penetration as a function of time, whereas the latter present a first slow and non-monotonous $\mathrm{Ga}$ penetration shortly followed by a very rapid 
increase of the $\mathrm{Ga}$ amount which has been interpreted as the decohesion of the material.

It appears that the stress clearly favours the Ga penetration, since when the Al polycrystal samples are fixed with stress the Ga penetration is more often observed, and with higher penetration rates. However so far, we have not been able to control this parameter. A controlled tensile device compatible with synchrotron micro-radiography is actually under design to follow the penetration under tensile stress both on bicrystals and on polycrystals. Experiments with better control of the stress are foreseen.

\section{Acknowledgements}

We wish to strongly thank P. Cloetens and E. Boller for their help during and after the syn- chrotron experiments, and Y. Brechet, E. Glickman and $\mathrm{K}$. Wolski for fruitful discussions.

\section{References}

[1] D.K. Clarke, M.L. Gee, in: D. Wolf, S. Yip (Eds.), Materials Interfaces, Chapman \& Hall, London, 1992, p. 255.

[2] B. Joseph, M. Picat, F. Barbier, Eur. Phys. J. AP 5 (1999) 19.

[3] E.E. Glickman, M. Nathan, J. Appl. Phys. 85 (1999) 3185.

[4] D. Chatain, E. Rabkin, J. Derenne, J. Bernardini, Acta. Mater. 49 (2001) 1123.

[5] J. Baruchel, J.Y. Buffiere, E. Maire, P. Merle, G. Peix, X-ray tomography in materials sience, Hermes, Paris, 2000.

[6] W. Ludwig, Ph.D. Thesis, Munich University (Germany), Development and Applications of Synchrotron Radiation Microtomography, 2001, unpublished.

[7] R.C. Hugo, R.G. Hoagland, Scri. Mater. 38 (1998) 523.

[8] N. Marié, K. Wolski, M. Biscondi, Scri. Mater. 43 (2001) 943. 